\title{
Structures pliées modulaires et architecture flexible
}

Modular Folding Structures and Flexible Architecture

Chloé Genevaux

\section{(2) OpenEdition}

1 Journals

Édition électronique

URL : https://journals.openedition.org/crau/591

DOI : $10.4000 /$ crau. 591

ISSN : 2547-5746

Éditeur

Éditions du patrimoine

Édition imprimée

Date de publication : 1 novembre 2012

Pagination : 242-248

ISBN : 978-2-7577-0108-9

ISSN : 1296-4077

Référence électronique

Chloé Genevaux, "Structures pliées modulaires et architecture flexible ", Les Cahiers de la recherche architecturale et urbaine [En ligne], 26/27 | 2012, mis en ligne le 01 novembre 2017, consulté le 21

septembre 2021. URL : http://journals.openedition.org/crau/591; DOI : https://doi.org/10.4000/crau. 591 
Le domaine du pliage est un sujet passionnant pour I'architecte, en ce qu'il associe " mise en forme et mobilité ». II s'agit ici d'aborder le pli sous l'angle des systèmes réticulés, à travers l'étude d'un principe modulaire dont l'élément de base, issu d'une simple bande pliée se refermant sur elle-même, est mobile et repliable. Les nombreuses possibilités d'assemblage permettent la genèse de formes variées, allant des figures géométriques "pures» aux formes dites «libres».

\section{Structures pliées modulaires et architecture flexible}

Le passage de l'imaginaire à la représentation est au cœur du processus de conception architecturale. L'outil informatique permet aujourd'hui de dessiner directement l'espace en trois dimensions et ainsi de représenter avec plus de facilité des projets aux géométries complexes. La CAO joue un rôle important dans l'engouement actuel des architectes pour les formes libres ${ }^{1}$. Cependant cet intérêt pour les espaces courbes n'est pas nouveau, et de nombreux architectes comme par exemple lannis Xenakis ${ }^{2}$, Jörg Utzon ou Frei Otto ont exploré cet univers sans I'aide de l'informatique. Au-delà de la fascination pour la forme extérieure, c'est la recherche de nouvelles relations entre l'homme et l'espace habité qui est au cœur de ce type de réflexions, à l'échelle du bâtiment mais aussi de la ville et du territoire.

\section{Pli et continuité}

Si le pli intéresse un nombre croissant d'architectes, c'est peut-être parce qu'au-delà de la forme, il peut être porteur de sens. L'ouvrage du philosophe Gilles Deleuze, Le pli, Leibniz et le baroque paru en $1988^{3}$

1. On désigne par forme libre, une forme ne pouvant être représentée uniquement par les traditionnels plans et coupes.

2. "Pour l'instant, le béton seul est à l'origine de l'architecture nouvelle du groupe volumétrique. II prépare le lit où les matières plastiques de demain formeront le fleuve riche de formes et de volumes que recèlent non seulement les être biologiques mais surtout les mathématiques les plus abstraites. Le système de références du corps humain ne sera plus l'angle droit et les surfaces planes, horizontales et verticales. Sa sensibilité se façonnera par un espace courbe. Du point de vue psychologique, c'est un enrichissement nouveau, géant, aux conséquences encore imprévisibles ", écrit lannis Xenakis en 1958 dans un article intitulé "Le Pavillon Philips à l'aube d'une architecture » (citation tirée du livre 
est cité en référence par de nombreux concepteurs, qui emploient le pli comme véritable langage architectural. Le pli, expression physique, a également une portée symbolique. Il permet notamment de relier les différentes parties d'un tout. C'est pourquoi en architecture, le pli est souvent synonyme de continuité, continuité des surfaces, continuité de fonctions, continuité entre l'intérieur et l'extérieur. En effet, la complexité issue du pli naît de l'unité (la feuille que l'on plie n'est pas divisée). Continuité que met déjà en avant Leibniz dans sa métaphore du pli : "La division du continu ne doit pas être considérée comme celle du sable en grains, mais comme celle d'une feuille de papier ou d'une tunique en plis, de telle façon qu'il puisse y avoir une infinité de plis, les uns plus petits que les autres, sans que le corps se dissolve jamais en points ou minima. »

L'architecture occidentale, longtemps basée sur la notion de fini avec la séparation nette entre l'intérieur et l'extérieur par d'épais murs de pierre ou de béton, évoluet-elle vers une expression plus mouvante faite de plis et de replis? II semble par ailleurs que notre conception de la réalité gagne, notamment avec le développement des réseaux globaux d'information, en continuité.

Le thème du pli sera abordé ici au travers d'une réflexion sur un système constructif expressif, issu de simples bandes pliées $^{4}$. Le pliage est employé comme outil géométrique pour la mise en forme de structures spatiales, de sorte que les surfaces générées par son intermédiaire expriment leur structuration interne. Le dessin des ombres et des arêtes marquées par cette géométrie confère à l'espace, une échelle, un rythme particulier. Ainsi, la notion de continuité, inclue dans la bande, favorise le tissage " organique » de relations intérieur-extérieur et la continuité des enveloppes. Cette typologie de formes libres échappe donc plus facilement à la " mollesse » que revêtent parfois les surfaces courbes et lisses.

\section{Bandes pliées pour structures légères Un principe d'économie de matière}

Le pli est un principe universel d'économie de matière, et donc d'énergie, essentiel dans le monde vivant. On le retrouve à de nombreuses échelles utiles pour I'homme: du microscopique pour certains matériaux, au macroscopique, par exemple la structure globale d'un bâtiment ou d'un ouvrage d'art. De nombreux végétaux ou animaux sont structurés par des plis. On distinguera deux principaux types d'emploi du pli dans le domaine des structures spatiales, la constitution de surfaces continues ou discontinues réticulées.

La structuration orientée d'une surface plane (continue) par des plis, comme c'est couramment le cas de nombreuses feuilles dans le monde végétal, est un principe que l'on retrouve dans de nombreux objets usuels comme par exemple la tôle ondulée, un matériau fin et léger qui tient sa rigidité du pli. Ainsi, le pliage permet de renforcer la résistance à la compression dans la direction perpendiculaire au pli, et à la traction dans la direction du pli. Ce travail de surface permet, en outre de rigidifier, de donner du volume et de façonner des formes en trois dimensions.

L'art de l'origami, source d'inspiration pour de nombreux architectes, a guidé la conception de bâtiments dont la structuration (spatiale, fonctionnelle ou simplement statique) est directement issue de

lannis Xenakis, Musique de l'architecture, présenté par Sharon Kanach, Marseille, Éditions Parenthèses, 2006).

3. Gilles Deleuze, Le pli, Leibniz et le baroque, Paris, Les Éditions de Minuit coll. "Critique », 1988.

4. Nous appelons bande, une plaque rectangulaire d'une longueur largement supérieure à sa largeur. 
ce travail de mise en forme. Des édifices extrêmement légers ont été construits suivant ce principe, par exemple certaines couvertures et façades aux portées impressionnantes réalisées par Pier Luigi Nervi ${ }^{5}$ ont une section n'excédant pas trois centimètres d'épaisseur. Plus récemment, la collaboration entre les architectes de Localarchitecture et Danilo Mondada, et Hani Buri et Yves Weinand chercheurs à l'IBOIs à l'École polytechnique fédérale de Lausanne, a conduit à la réalisation $d^{\prime}$ 'une chapelle en bois entièrement plissée ${ }^{6}$. Dans ce projet exemplaire, outre sa contribution à la structure du bâtiment, le pli confère à l'espace un caractère expressif et sculptural.

Le pliage peut également intervenir dans le domaine des structures spatiales réticulées, pour lesquelles la question des assemblages est essentielle. Ici, la conception du " nœud » assurant la connexion entre les barres a une incidence considérable sur le poids, le coût, le temps de montage, le comportement mécanique et l'esthétique du projet. Cette " connectique » a fait l'objet de nombreuses recherches, puisant pour la plupart dans le répertoire géométrique des polyèdres. Les systèmes basés sur des géométries polyédriques offrent une liberté limitée aux concepteurs. La réalisation sur-mesure (percement de nœuds sphériques aux emplacements requis par exemple) est aujourd'hui économiquement viable grâce à l'emploi de machines à commande numérique. On confond pourtant parfois le mode de représentation d'une géométrie (points segments) et sa matérialisation au final ; ainsi la notion même de conception par nœuds et barres peut être remise en question, comme le propose
I'architecte-ingénieur Yves Weinand dans un article intitulé "Construire sans Nœuds" ". II suggère que la structure peut être pensée non pas comme une maille au sein de laquelle le nœud reprend tous les efforts, mais comme une juxtaposition de triangles indépendants. En ce sens, l'emploi de bandes pliées retourne complètement notre façon de concevoir le nœud: les bandes continues sont directement assemblées entreelles, les nœuds n'étant plus des pièces coûteuses fabriquées indépendamment mais le résultat direct de l'assemblage des éléments constitutifs de la structure. Ici, barres et nœuds ne sont plus distincts mais continus.

Outre un façonnage simplifié, l'emploi de bandes rectilignes réduit d'autant plus le coût de fabrication qu'il il ne produit pratiquement aucune chute, l'économie de matière est donc maximale. La conception de structures à partir de bandes pliées peut donc constituer une solution économique à la réalisation d'architectures de formes libres. En effet, même si les technologies de fabrication assistée par ordinateur se développent, le passage à la réalisation reste une phase critique. De manière générale, Lorsque une forme est conçue au départ sans prise en compte du mode constructif, la complexité et la multiplicité des éléments de construction augmentent considérablement le coût et les temps de réalisation.

\section{Familles de bandes pliées Vers un système modulaire}

Le domaine des bandes pliées est vaste. On peut distinguer deux grandes familles de bandes pliées, les bandes « ouvertes » et les bandes "fermées » c'est-à-
5. Entrepôt expérimental en ferrociment, Rome, 1946. Grande Halle du palais d'exposition de Turin, 1947-1949.

6. Chapelle, Hôpital de Saint-Loup, Suisse, Localarchitecture et Danilo Mondada (architectes), IBOIs, Hani Buri et Yves Weinand (étude structure), 2008
7. «Curieusement l'ingénieur civil n'a que très rarement remis en question le principe du nœud. Dans I'histoire de la structure métallique, il est intéressant de constater que l'expression constructive du nœud est clairement cataloguée. On part d'un élément sur lequel on vient fixer les barres d'un treillis, par exemple. Classiquement, il s'agit d'un gousset ou d'un plat, cela peut prendre la forme d'une sphère ou d'un crampon mais il existe finalement toujours un élément physique incarnant le nœud. Ces nœuds ont toujours joué un rôle important et... problématique, puisque le nœud correspond souvent à une zone affaiblie de la section courante. Une conception permettant d'éviter purement et simplement le nœud mérite donc d'être examinée. À Lommel, on assemble de simples triangles. Le point de départ est un triangle (ou une multitude de triangles) et non pas une maille. Localement, la matière n'est pas continue à l'endroit des nœuds (puisqu'il s'agit d'une multitude de triangles 

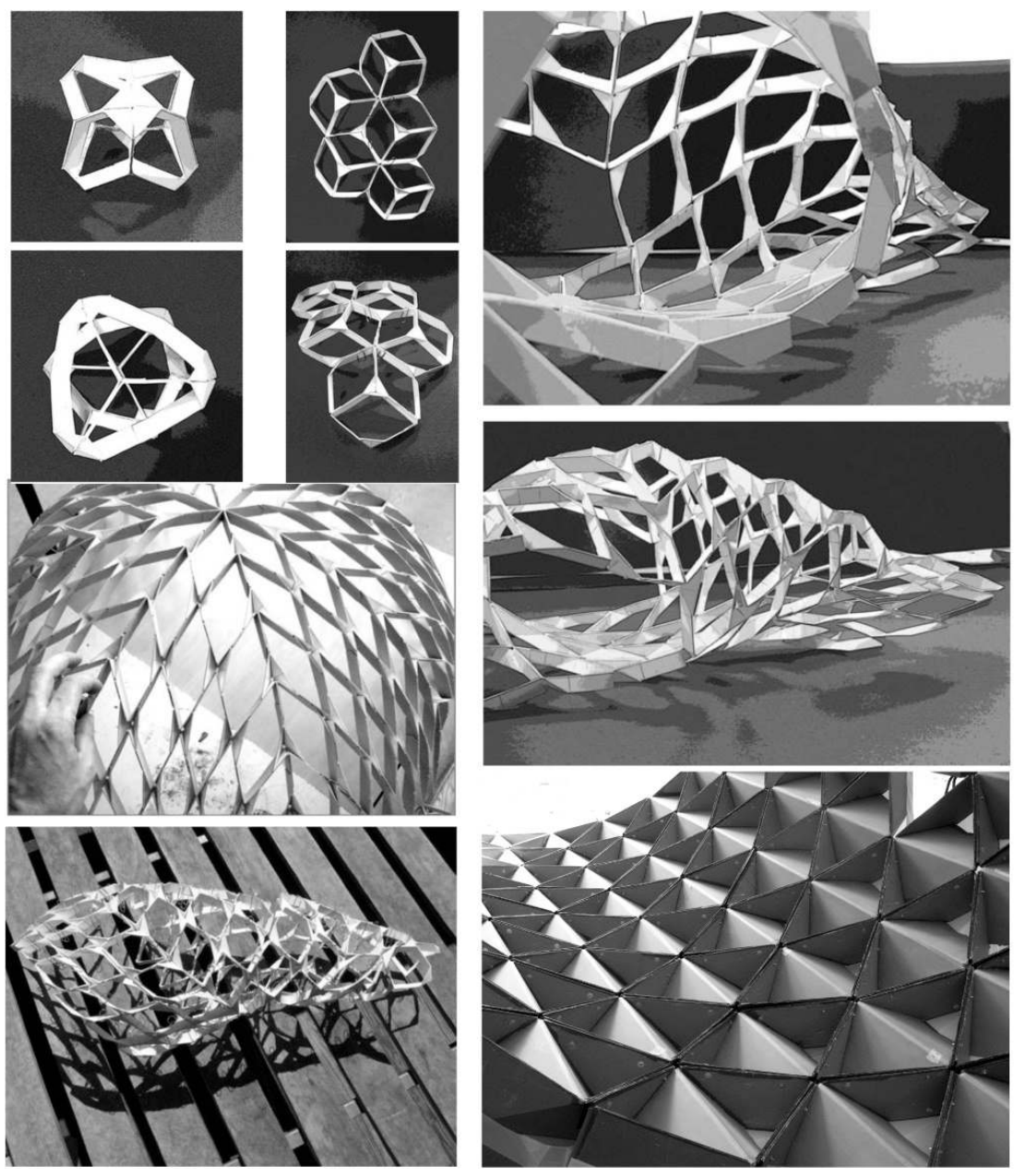

d'un même élément modulaire formé d'une simple bande pliée. Modèle déposé.
Maquettes et structures légères éphémères réalisées avec le collectif Archiwaste à partir
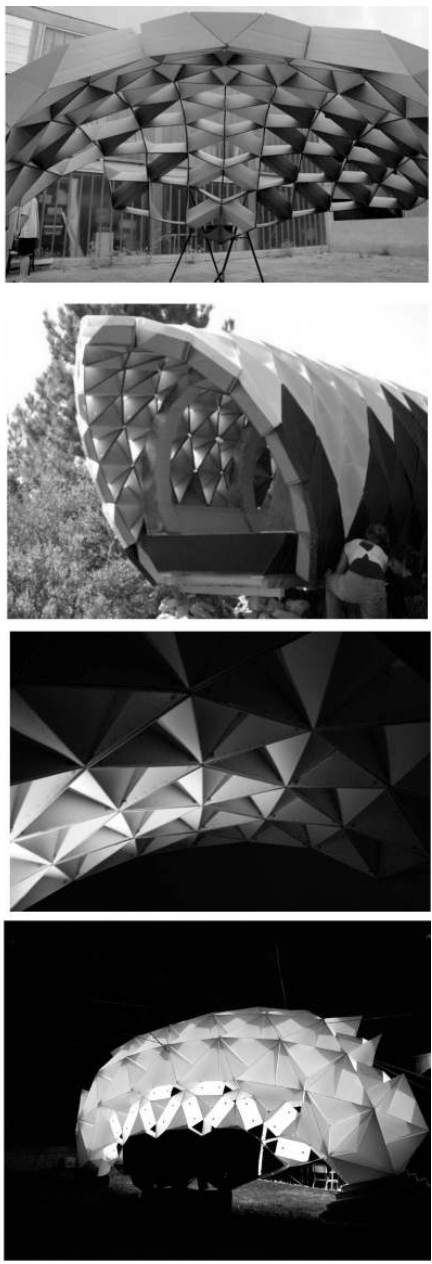

indépendants). La matière d'un nœud classique est donc supprimée au profit d'un fonctionnement structurel surfacique dans l'espace. "Yves Weinand, "Construire sans nœuds ", revue Tracés n 15/16, Lausanne, août 2008, p. 24-28. 
dire formant une chaîne fermée sur elle-même. Ces deux familles de bandes permettent la mise en forme de surfaces issues de polyèdres ou de surfaces à simple et double courbure.

\section{Les bandes ouvertes}

On appelle ici bandes « ouvertes » les bandes pliées ne se refermant pas sur elles-mêmes. Leur degré de liberté dépend du nombre de plis. Lorsque ces plis convergent en un point, le produit simultané des pliages équivaut à une rotation autour du point d'intersection de plis et correspond mécaniquement à une rotule spatiale. Lorsque les plis convergent en plusieurs points, ces points correspondent alors à divers pôles d'articulation. Les bandes ouvertes conservent leur mobilité tant qu'elles ne sont pas refermées, en composant des triangles, ou par de nœuds indéformables formant des trièdres. On peut également leur ajouter le nombre d'éléments nécessaires à leur stabilisation. Certaines conditions géométriques sont nécessaires pour que la bande se referme. Selon I'orientation des plis, la bande peut former un polygone convexe ou concave, dont les bords sont coplanaires ou non. Les bandes fermées peuvent être divisées en deux catégories : les bandes non déformables, et les bandes déformables qui conservent une mobilité.

\section{Les bandes fermées non déformables}

Si l'on prolonge les plis jusqu'à leur point de rencontre, on remarque que l'on peut considérer la bande comme une section d'un volume plus grand. Si celui-ci est indéformable (constitué de triangles), la bande sera indéformable également. Si le volume dont elle est issue est déformable, la mobilité de la bande sera permise.

\section{Les bandes fermées déformables}

Les bandes déformables sont des chaînes cinématiques fermées. Ce domaine est exploré par les ingénieurs mais peu d'architectes s'y sont intéressés. Les chaînes cinématiques fermées sont employées dans le bâtiment pour la réalisation de structures mobiles. Les structures déployables sont des structures qui peuvent être transportées "pliées ", occupant alors un minimum d'espace, et dépliées sur site. Les structures déployables sont développées pour l'aérospatiale, comme les satellites par exemple. Contenues dans un emballage le plus petit possible pour le transport, elles doivent ensuite se déployer dans l'espace. Mais les structures mobiles sont également utilisées dans la construction de grandes couvertures rétractables, de stades par exemple, ou pour des constructions légères itinérantes.

Les caractéristiques recherchées alors sont la repliabilité en un minimum d'espace, un élément composé de mécanismes simples pouvant être assemblé pour créer de grandes structures, et la facilité de blocage de la mobilité du système (on retient alors plutôt les mécanismes a un seul degré de liberté). Dans le processus, il faut également s'assurer de la compatibilité géométrique de l'ensemble, afin de permettre le mouvement. La recherche dans ce domaine des trente dernières années s'est concentrée sur la construction de mécanismes plans. Les modules de construction comprennent un ou plusieurs types de mécanismes 
plans avec un degré de liberté. Les éléments sont ensuite assemblés de sorte à ce que les conditions de compatibilité géométrique soient rencontrées. Les mécanismes 3D sont rarement employés, probablement du fait de la difficulté mathématique de définition des conditions de compatibilité. Le mécanisme le plus simple est le losange. Dans son travail de thèse sur les structures pliables ${ }^{8}$, Santiago Calatrava a étudié le mécanisme du losange à un degré de liberté et les assemblages possibles pour la réalisation de structures mobiles aux formes polyhédrales.

Si l'objectif n'est pas la réalisation de structures entièrement pliables mais uniquement d'éléments constructifs que l'on plie à plat pour le transport et déplie pour l'assemblage sur site, la liberté formelle est alors plus grande. L'emploi de bandes continues que l'on plie permet de concevoir des chaînes cinématiques fermées réalisées simplement. Les losanges non plus plans mais gauches, formés par des bandes pliées, peuvent mener à la mise au point de systèmes constructifs économiques pour la réalisation de formes courbes. La variable cinématique permet une modification du gauchissement du losange et donc de varier la courbure des surfaces générées.

La recherche d'un élément modulaire a pour objectif de trouver un équilibre entre composants standards et pièces réalisés sur-mesure. Le système modulaire étudié est caractérisé par l'assemblage d'éléments identiques dans leur géométrie mais variables de par leur cinématique. Le principe est d'assembler un maximum d'éléments identiques pour générer des familles de formes variées.

\section{Processus de morphogenèse}

Le pli, outre la notion de continuité, contient intrinsèquement l'acte de transformation. L'action de plier est la transformation topologique d'un élément, c'est un moyen de sculpter la matière. Le pli est donc à la fois processus de mise en mouvement et de mise en forme. Or il semble que les processus de génération formels prennent de plus en plus d'importance dans l'imaginaire conceptuel des architectes. Le bâtiment est dès lors pensé non plus comme un objet fini régis par des paramètres fonctionnels parfois restrictifs mais comme une étape de stabilisation dans la genèse et la transformation d'une forme. II est intéressant de constater que cette manière de concevoir, encore une fois, tend à introduire la notion d'infini dans l'architecture. Cette conception de la genèse d'une forme est fortement inspirée du domaine de la biologie. La biologie aborde la question de la forme notamment à travers l'étude de la morphogenèse, c'est-à-dire la manière dont les formes naissent et se modifient. La morphogenèse naturelle, en relation avec les contraintes extrinsèques et les stimuli de l'environnement, est un processus au sein duquel forme et matière sont indissociables, à toutes les échelles.

Dans la nature, la compréhension d'une forme passe par la compréhension de son processus de morphogenèse. De même, la compréhension des formes générées par assemblage d'éléments pliés (bandes fermées déformables) passe par la compréhension des lois et contraintes d'assemblage issues de la géométrie de la cellule même. Par analogie avec le monde vivant, le terme " cellule » désigne ici l'élément modulaire 
plié. Cette "cellule » dont on connaît les caractéristiques géométriques, cinématiques et physiques est le point de départ d'un système constructif modulaire révolutionnaire. Plusieurs cellules assemblées peuvent générer des formes diverses, composées de surfaces à simple ou double courbure. Ceci pose la question de la genèse d'un forme par prolifération de cellules. Comment dès lors prévoir et maîtriser la géométrie de la forme globale lorsque l'on part d'un élément local ? Quelle peut être l'influence des contraintes extérieures sur la morphogenèse ? Et comment dès lors optimiser ce processus de la conception à sa matérialisation?

La biologie et l'informatique joueront vraisemblablement un rôle important dans la conception d'un outil de modélisation " intelligent » des structures spatiales réticulées construites à partir de tels éléments modulaires.

L'architecture assistée par l'informatique peut s'enrichir de concepts issus de la biologie évolutionnaire. Certains algorithmes, pensés en analogie avec la génétique, permettent d'imiter un processus d'évolution inhérent au vivant : une population de base est générée aléatoirement par la machine en fonction d'un ensemble de paramètres déterminés au départ (par exemple la course du soleil, un prospect, ou les données simplifiées du programme), on effectue ensuite une sélection puis des croisements entre individus sélectionnés et enfin des mutations. Le principe est d'effectuer ces opérations un maximum de fois afin d'augmenter la justesse du résultat. Aujourd'hui, certains architectes s'emparent de ce processus de simulation d'évolution mis en place par des algorithmes génétiques pour expérimenter des générations de solutions architecturales en réponses à des contraintes données ${ }^{9}$. Les critères et le mode de sélection, automatique ou effectué par le concepteur, est alors primordial pour la qualité des résultats. C'est bien le processus qui crée la forme qui importe dans ces recherches. L'objectif n'est certainement pas la conception effectuée par ordinateur mais une aide à l'exploration de solutions. II est inhérent à l'architecture de connecter différentes disciplines : la mécanique et la cinématique, la structure et la résistance des matériaux, la programmation informatique, la géométrie, sont autant domaines essentiels à l'avancée de ce travail.

II incombe au concepteur de définir une logique préalable, des objectifs, et une éthique, afin d'acquérir une compréhension globale de cette "forme-processus ", et ainsi de pouvoir collaborer efficacement avec les professionnels d'autres disciplines au développement et à la mise en œuvre de systèmes constructifs innovants.

Imaginez un instant, une architecture guidée par I'humain, se développant à la manière d'un organisme vivant, orientée vers la lumière et profondément enracinée dans la terre... pour qu'au $\mathrm{xx}^{\mathrm{e}}$ siècle, enfin détaché de l'archaïsme et des superstitions du passé, un nouvel état d'esprit d'habiter voie le jour.
9. Philippe Marin a soutenu, le $31 \mathrm{mai}$ 2010, une thèse de doctorat en Sciences de l'architecture, sous la direction de Jean-Claude Bignon, Exploration des mécanismes évolutionnaires appliqués à la conception architecturale. Mise en œuvre d'un algorithme génétique guidé par les qualités solaires passives de l'enveloppe. 